\title{
Fractal Analysis of the Helix Nebula (NGC 7293)
}

\author{
Srabani Datta \\ Department of Applied Mathematics, University College of Science \& \\ Technology, University of Calcutta, 92 A.P.C. Road, Calcutta 700 009, \\ India
}

\begin{abstract}
In this paper, the Helix nebula (NGC 7293) is postulated to be a fractal describable by a Julia set. In order to support this hypothesis, a cometary filament is chosen from the periphery of the shell and is analyzed using an automated software. The box dimension of NGC 7293 is found to be 1.841059 .
\end{abstract}

\section{Introduction}

The Helix nebula is a Type I planetary nebula (PN) in the constellation Aquarius at a distance of $160 \mathrm{pc}$ from us (Meaburn et al. 1998). The Helix derived its name from the shape of its toroidal cloud structure around a central star. This ring, which may be an open structure, is at a distance of about $200^{\prime \prime}$ from the central star and is tilted at an angle of $14^{\circ}$ to the plane of the sky. The inner part of this ring is seen to expand at a velocity of $20 \mathrm{~km} / \mathrm{sec}$. This ring is in turn surrounded by a massive molecular envelope. The central star of the Helix is a white dwarf of mass $0.93 \mathrm{M}_{\odot}$ and effective temperature $120000 \mathrm{~K}$ (Henry et al. 1999). The central star has ionized the ring of the nebula, but this ionization is not uniform. It is stronger around the SSW and NNW parts, producing a thinner ionization front at these locations. Finally, a low-ionization filamentary arc is found to form a halo-like structure to the whole complex.

\section{Method}

In this paper, the author investigates the structure of the Helix nebula with the aim of explaining the general morphology of PNs in the light of the Julia set (see fig. 1 of Datta 2001a) arising from the non-linear function

$$
f(z)=z^{2}+c, c=-0.745429+0.113008 i
$$

where both $z$ and $c$ are complex quantities. This set is a fractal (Falconer 1997) and its dimension is measured by an automated fractal dimension analysis software, Benoit 1.3, procured from Trusoft International Inc., USA (Seffens 1999). For the analysis, a set of 10 measurements were taken of a filament from a HST WFPC2 image (O'Dell \& Handron 1996). The method is described in Datta (2001a). 


\section{Discussion}

Current models of PN structure formation (Goncalves, Corradi \& Mampaso 2001) fail to account for all aspects of PN morphology. However, the fractal model does not have any of the drawbacks of the older ones. Fast Low Ionization Emission Regions (FLIERS), Bipolar Rotating Episodic jeTs (BRETS), filaments, symmetrical jets, pairs of knots, low-ionization structures can easily be explained by chaotic dynamics arising from turbulence in the interstellar medium. Studies by Elmegreen (1999), Datta (2001a, b) and references therein, have conclusively shown that this process gives rise to the hierarchical structure of molecular clouds (Larson 1981) and the stars in them (Larson 1995). Therefore, the post Asymptotic Giant Branch (AGB) star wind can only cause to push back the already existing structure around it. The existence of jet-like structures of age younger than the PN and Low Ionization Structures (LIS) irrespective of type are pointers to this fact. However, detailed studies will have to be done in order to determine the truth.

Acknowledgments. The author wishes to thank her guide, Prof. B. Basu, former head of department, Department of Applied Mathematics, University College of Science \& Technology, University of Calcutta; Dr. N.K. Dey, Ramakrishna Mission Residential College, Narendrapur, Calcutta; Dr.D. Mitra \& Prof. R. Gupta, IUCAA, Pune, India. Thanks also goes to Dr. M. Hart, Department of Pure Mathematics \& Dr. C. Tadhunter, Department of Physics \& Astronomy, University of Sheffield, U.K.

\section{References}

Datta, S. 2001a, in Automated Data Analysis in Astronomy , ed. R.Gupta , H.P. Singh \& C.A.L. Bailer-Jones, (New Delhi :Narosa), 253

Datta, S. 2001b , Bulletin of the Calcutta Mathematical Society, 93

Elmegreen, B.G. 1999 , Ap.J. , 527, 226

Falconer, K. 1997, Fractal Geometry, Wiley \& Sons, Chichester

Goncalves, D.R., Corradi, R.L.M., \& Mampaso, A. 2001, Ap.J. , 547, 302

Henry, R.B.C. et al. 1999 Ap.J. , 517, 782

Larson, R.B. 1981 MNRAS , 194, 809

Larson, R.B. 1995 MNRAS, 272, 213

Meaburn, J. et al. 1998 MNRAS, 294, 201

O'Dell, C.R. \& Handron 1996 A.J., 111, 1630

Seffens, W. 1999 Science, 285, 1228 